\title{
¿Reinas de la casa? \\ Las mujeres y el hogar frente a la pandemia actual
}

\section{Queens of the house? Women and the home in the face of current pandemic}

Libia Yuritzi Contreras Yttesen Universidad Nacional Autónoma de México libia.contrerasy@derecho.unam.mx

\section{Resumen}

El siguiente trabajo se propone analizar la relación entre las mujeres y el hogar, entendiendo a éste como un ámbito de lo privado, que a la luz las teorizaciones del llamado feminismo académico del siglo $\mathrm{XX}$, permite retomar la discusión sobre la dicotomía entre lo público y lo privado; en este caso presentando al hogar como un espacio de la vida privada que, tras evidenciar las condiciones de violencia que viven las mujeres en estos 
espacios, hace más patente un viejo reclamo del feminismo de la segunda ola sobre hacer visible lo privado del hogar como preocupación pública. Lo anterior cobra relevancia si atisbamos el contexto actual debido al periodo de contingencia sanitaria por COVID-19 y la promoción de la campaña nacional «Quédate en casa» en México.

Palabras clave: Género, Mujeres, Hogar, Pandemia, COVID-19.

\section{Abstract}

The next paper aims to analyze the relationship between women and their home, understanding 'home' as a private space which based on the s. XX academic feminism theory, this allows us to approach the dichotomy between public and private discussion. In this case, we present home as a space of private life that, after highlighting the conditions of violence that women experience in these spaces, brings an old claim of feminism Second-wave about making home visible as something of public concern. Previous words become relevant if we notice current context of pandemic due to COVID-19 and the promotion of the national campaign «Stay at home» in Mexico.

Key words: Gender, Women, Home, Pandemic, COVID19.

Como bien señalan Estela Serret y Jessica Méndez Mercado, una creencia errónea bastante frecuente dicta que 
el feminismo surge en la década de 1960 al calor de los movimientos sociales de la época, no obstante, siguiendo a las autoras, las primeras aportaciones feministas documentadas datan del siglo XVII, concretamente en 1622 con la obra De la igualdad entre los hombres y las mujeres, escrita por Marie Le Jars de Gournay (Serret y Méndez 2011; 12). Esta filósofa francesa fue contemporánea de los teóricos partidarios de la incipiente corriente racionalista presente en Europa durante los siglos XVI y XVII, quienes afirmaban la autonomía y la independencia de la razón humana frente a la razón teológica imperante durante la Edad Media, lo cual cabe destacar que fue el basamento de la filosofía política iusnaturalista posterior (Asuástegui et al 1998; 575). Marie de Gournay se sorprende de que los pensadores de aquella época cuestionen, por un lado, la legitimidad del régimen político estamental del feudalismo argumentando la igualdad natural entre todas las personas con base en su capacidad racional y que, por otro lado, a pesar de proponer una universalización de la idea de un individuo racional como atributo de todos los seres con discernimiento cognitivo y moral, no se inmutaron al afirmar a las mujeres como subordinadas a la tutela masculina y excluirlas del proyecto igualitarista y liberador no sólo respecto al acceso al poder político y al espacio público, sino también marginándolas del plano educativo y de gran parte de los derechos civiles que se defendieron más tarde (Serret y Méndez 2011; 13). 
Así, el feminismo nace ante la constante negación de la autonomía de la mujer, constituyéndose como una protesta que lucha contra las incongruencias del periodo de la Ilustración europea, por ende, este feminismo emergente puede considerarse como un movimiento intelectual crítico de corte ilustrado. Posteriormente, y haciendo un brinco histórico bastante arbitrario, las consecuencias epistemológicas y políticas pasan a su desarrollo como movimiento social con el sufragismo del siglo XIX hasta su posterior irrupción en la academia y la investigación en el siglo $\mathrm{XX}$, con lo que algunas autoras denominan como 'feminismo académico' (Serret y Méndez 2011; 15).

De hecho, uno de los puntos clave para entender el rumbo que ha tomado la filosofía feminista tras su aparición en la vida académica universitaria ha sido a través de la recuperación de la filosofía hecha por mujeres o sobre mujeres, además de elaborar ejercicios críticos e incluir estudios sobre el histórico androcentrismo de la filosofía. Entre tanto, las autoras coinciden en que puede afirmarse que en un principio estos estudios, más que una teoría, representaban una perspectiva crítica, de manera que el mérito de las aportaciones feministas fue realizar una labor interpretativa de teorizaciones del pasado pero desde un enfoque feminista: «La reflexión feminista no se plantea en principio como una teoría sino como una perspectiva crítica que devela un problema siempre presente, pero siempre ocultado» (Serret y Méndez 2011; 41). En consecuencia, el 
feminismo académico que florece a mediados del siglo $\mathrm{XX}$ representa el campo en el cual se introduce la noción de género como categoría de análisis político y social, y éste es el punto desde donde se desarrollará el análisis del presente trabajo.

Actualmente, al igual que con el concepto anglosajón gender, la correspondiente acepción del español sobre género tiene una referencia predominantemente cultural. El feminismo de mediados de siglo XX hace una contribución teórica marcando la diferencia entre lo biológico o anatómico (sexo) frente a la construcción imaginario-social de 'lo femenino' y 'lo masculino' (género). Señalar esta diferencia le permitió al feminismo mostrar la forma en la que se ha construido culturalmente una estructura binaria (hombre-mujer) que crea y reproduce un orden social con valores determinados para los hombres y para las mujeres, el cual va más allá de esencias o naturalizaciones que se deriven de cierta biología. En nuestra cultura, pensar en los atributos del rol masculino como cabal, valiente, fuerte, agresivo, impulsivo, racional, insensible e independiente, o bien, en las características de lo femenino como pasiva, débil, sumisa, dependiente, sentimental, afectiva, muestra sólo algunos indicios sobre cómo se han asumido históricamente estereotipos de género basados en cierto orden social atravesado por el género.

Marta Lamas afirma que la crítica feminista empezó a interrogar sobre las formas en las que el cuerpo es percibido 
en un entorno social estructurado por el género. En palabras de la autora: «El género se conceptualizó como el conjunto de ideas, representaciones, prácticas y prescripciones sociales que una cultura desarrolla desde la diferencia anatómica entre hombres y mujeres, para simbolizar y construir socialmente lo que es "propio" de los hombres y "propio" de las mujeres» (Lamas 2000; 2). Así, el género puede entenderse como un elemento fundamental en la construcción de la cultura y, por ende, en la configuración identitaria.

La noción de género muestra cómo a través de la circunstancia biológica del sexo de la persona, socialmente se atribuyen características, actividades $\mathrm{y}$, además, se jerarquiza a los roles sociales (hombres/mujeres) de manera inequitativa, es decir, bajo un esquema de distribución desigual del poder en el que, por lo regular, se inferioriza a las mujeres por su condición biológica: las mujeres son más débiles que los hombres por su constitución anatómica, por ende son más delicadas, razón por la cual deben estar en casa, o bien, los hombres son más inteligentes que las mujeres porque tienen el cerebro más grande, por lo tanto son mejores para tomar decisiones y gobernar, sólo por mencionar algunos ejemplos. En otras palabras, se hace evidente un imaginario social de género que marca ciertas reglas y mandatos de género: «las mujeres no deberían comportarse de tal manera», «los hombres no lloran», etc. De hecho, algunas de las ventajas que trae consigo esta 
noción de género es que nos permite cuestionar relaciones de desigualdad, mandatos sociales y también nos posibilita el evidenciar dinámicas de género que permanecían invisibilizadas o que se asumían como naturales; es por lo anterior que la conceptualización del género como categoría tiene una fuerte carga política (Saldarriaga y Gómez 2018; 46).

En consecuencia, paulatinamente se usó la noción y la categoría género en diversos campos de conocimientos con la finalidad de develar prácticas, sistemas e instituciones sociales en las que subsisten formas de dominación/subordinación, o bien naturalizaciones, que justifican culturalmente la discriminación y la violencia de género: «Los sistemas de género, sin importar su periodo histórico, son sistemas binarios que oponen el hombre a la mujer, lo masculino a lo femenino, y esto, por lo general, no en un plan de igualdad sino en un orden jerárquico» (Conway 2015; 32). Algunos ejemplos dentro de ese esquema de dominación y de violencia contra las mujeres pueden evidenciarse cuando notamos lo tardío que ha sido el reconocimiento de los derechos de las mujeres en cuanto a educación, sufragio universal, derechos laborales, etc.

De hecho, para tratar de analizar la naturaleza de la violencia de género podríamos recurrir a la teoría del triángulo de la violencia del sociólogo noruego Johan Galtung, quien nos presenta tres dimensiones de la violencia en las sociedades contemporáneas: la primera, la 
violencia directa, que puede ser la violencia física o psicológica. La segunda, la violencia indirecta, que es en la que no existe emisor personal identificable; aquí destaca por ejemplo la pobreza como un tipo violencia indirecta. Finalmente, la tercera forma de violencia es la simbólica o cultural, la cual comprende aquellas formas de daño que se expresan en las mentalidades, creencias y valores (Galtung 2003; 20), de modo que un ejemplo de este tipo de violencia es, por supuesto, la violencia de género.

Con todo, es necesario remarcar que, a pesar de que la reformulación de la noción de género se atribuye al feminismo académico de mediados del siglo $\mathrm{XX}$, discurrir sobre violencia de género no alude exclusivamente a violencia contra las mujeres, ya que pensar el género como categoría de análisis nos permite vislumbrar más violencias relacionadas con el género, por ejemplo, aquellas basadas en estereotipos que también afectan a otras manifestaciones identitarias. De hecho, este tipo de violencia también incluye aquellas basadas en la discriminación por orientación sexual o identidad de género, o bien, las violencias que se ejercen sobre las masculinidades disidentes. Empero, más allá de esta aclaración, a continuación nos enfocaremos casi de manera exclusiva en examinar la violencia contra las mujeres en un espacio privado como es el hogar.

Gayle Rubín es una de las académicas que destaca el papel que ha desempeñado el género en la división sexual 
del trabajo (Fernández 1998). En realidad, fue hasta décadas recientes que muchas mujeres comenzaron a introducirse en el mundo laboral, no obstante, si nos preguntáramos dónde estaban antes las mujeres, probablemente la respuesta sería que ellas estaban en la esfera de lo privado: en los hogares ${ }^{1}$. Si bien, definir con claridad la diferencia entre lo público y lo privado es una tarea complicada ya que son áreas que constantemente se interceptan o se confunden, como bien señala Yamile Delgado de Smith, podríamos afirmar que: «tradicionalmente se relaciona lo público con lo político y lo privado con lo doméstico» (Delgado 2008; 118). En ese sentido, diversas autoras explican que lo masculino y el ser hombre aparece vinculado con el ámbito público: «En ese espacio público se espera que el hombre ostente sabiduría, poder, ejercicio del dominio y demuestre su excelencia y eficacia, su racionalidad. Este espacio es visible, tangible, es el único en donde el trabajo es remunerado, medible» (Delgado 2008; 116).

Mientras, lo que se ha asignado culturalmente a la mujer, a lo femenino, se ubica en el ámbito privado, doméstico y familiar, el cual resulta un espacio para el cuidado, la reproducción de la vida y la atención a los otros a través de un trabajo no remunerado e invisibilizado. En

${ }^{1}$ Para la redacción de este trabajo tomaré como sinónimos hogar (el hogar) y casa (la casa); entendiendo a ambos como espacios que, en general, se han entendido como parte de la esfera de lo privado. 
vista de este escenario, no es de extrañar que las mujeres hayan estado marginadas de la vida pública durante siglos; lo curioso es que esta exclusión de las mujeres de la vida social se ha justificado frecuentemente con argumentos naturalistas que advierten más bien un desinterés y una incompatibilidad de las mujeres con la política debido a una supuesta vocación maternal y a una orientación particularista (Delgado 2008; 117).

Por ser la mujer quien está mejor dotada para el ámbito de lo privado y las exigencias que de éste se derivan, es que se busca evitar históricamente el acceso al trabajo, a la educación, a la ciencia y desde luego a las esferas de poder y toma de decisiones. En consecuencia, la participación de la mujer en los procesos sociales y políticos sigue bloqueada por una especie de «androcracia», que ha alimentado la creencia de que el mundo de lo público es privativo de los varones (Delgado 2008; 117).

De este modo, podemos notar cómo el orden de género crea y estratifica ese binarismo entre lo masculino y lo femenino, dejando al espacio público como un espacio predominantemente masculino, mientras el ámbito privado, el del hogar, se percibe como un espacio feminizado que enmarca a las mujeres como figuras de la domesticidad: 'las reinas de la casa'. 
Tal como apunta el filósofo Michael Warner, el feminismo de la segunda ola se centró precisamente en la crítica sobre lo público y lo privado, señalando que lo masculino era a lo público como lo femenino era a lo privado: «la caracterización por género de lo público y lo privado contribuiría a explicar transculturalmente la subordinación de las mujeres. En este contexto, privado significa espacios y funciones domésticos, y público hace referencia a contextos en los cuales los hombres hablan y toman decisiones por la comunidad» (Warner 2012; 30). Siguiendo al autor, esta discusión fue central para casi dos siglos de escritos feministas y de lucha política.

Como consecuencia, en el movimiento y pensamiento feminista persistió una gran insistencia en visibilizar las cuestiones domésticas y privadas como un área de preocupación común y de necesaria intervención estatal en casos como la violación marital, el maltrato del cónyuge, la prostitución, el aborto, etc. En palabras de Catharine MacKinnon: «Para las mujeres la medida de la intimidad ha sido la medida de la opresión. Por eso el feminismo tuvo que hacer explotar lo privado. Por eso el feminismo ha visto lo personal como lo político. Lo privado es lo público para aquellos para quienes lo personal es lo político» (MacKinnon 1987; 100).

Ciertamente, lo anterior ya nos permite vislumbrar lo que se mencionaba sobre la complejidad de distinguir de manera clara y distinta lo público de lo privado. Siguiendo 
a Warner, estas definiciones parecen involucrar confusiones y hasta contradicciones ya que es habitual que se entremezclen ideas diferentes sobre la esfera pública y la privada: «Una conversación privada puede tener lugar en un foro público, una recámara puede ser un espacio público y comercial, como en un hotel» (Warner 2012; 25), es decir, los conceptos se traslapan en razón de que la mayoría de las cosas son privadas en un sentido y públicas en otro.

Históricamente, de acuerdo con el pensamiento romano y su repercusión en el medievo, la distinción entre lo público y lo privado hacía referencia a lugares o espacios, es decir, era notoria una distinción en sentido físico-espacial (Warner 2012; 24); mientras, la cultura moderna comienza a aludir más bien a relaciones que a espacios, por ejemplo: la autonomía, la autodeterminación o la privacidad. Por si fuera poco, desde el punto de vista conceptual, Warner señala que estos términos suelen definirse uno en oposición al otro, luego lo público y lo privado compiten, y otras veces se complementan (Warner 2012; 25). En resumen, definir claramente lo público y lo privado es una cuestión que ha variado históricamente y que dependerá de la postura analítica desde la que nos aproximemos. Con todo, lo que el autor quiere recalcar es que la insistencia de los movimientos feministas por transformar la sexualidad o el género son cuestionamientos públicos de la vida privada, lo que a la par supone un problema de lo público y lo privado en su propia práctica, es decir, se trata de movimientos 
públicos que cuestionan asuntos privados e íntimos (Warner 2012; 29).

Tras la ambigüedad que deja la confusa dicotomía entre lo público y lo privado, otras narrativas se presentan en la modernidad, por ejemplo, la privacidad va adquiriendo un valor propio y distintivo con cualidades como libertad, individualidad, interioridad y autenticidad. De hecho, tampoco es de sorprender que, aunado al éxito de la ideología liberal y al progreso de las sociedades capitalistas, detrás de la noción moderna de propiedad privada subsista también una afrenta contra el Estado, la cual se nos ha presentado como aquella que trata de poner límites al poder público y se propone ensanchar la libertad individual.

Incluso, como Warner señala, en el mundo jurídico existen variedad de contextos y reflexiones sobre la privacidad (desde el derecho constitucional, el derecho familiar o el derecho de la propiedad) (Warner 2012; 28). En efecto, el derecho a la privacidad puede vincularse también con el derecho a formar relaciones íntimas, con la orientación y la identidad sexual, es decir, con los derechos vinculados a la libertad personal que, al mismo tiempo, entrañan diferentes imaginarios sobre el cuerpo y la configuración de la identidad. En ese orden de ideas, esta concepción moderna sobre lo público y lo privado también nos invita a pensar al hogar como un espacio privado que permite que florezca la libertad personal, la seguridad, el 
confort y la salud. Sin embargo, si bien es cierto que la casa nos puede proveer de ciertas comodidades, también representa un espacio digno de análisis desde un enfoque contemporáneo y con perspectiva de género, sobre todo, desde un estudio que no pierda de vista las circunstancias actuales de confinamiento debido a la contingencia sanitaria por SARS CoV-2 (COVID-19).

Tal como se señalaba anteriormente, en la actualidad muchas mujeres cuentan con un empleo, sin embargo, su vida en el hogar suele conllevar una doble o triple jornada de trabajo, lo cual quiere decir que ellas, además de contar con un empleo, deben llegar a casa y «cumplir los deberes», con poca o nula «ayuda», sin remuneración y con escaso reconocimiento (Foro Jurídico, 27 de noviembre de 2020). Adicionalmente, ya explicábamos que se ha normalizado que las actividades femeninas se vinculen con trabajos de cuidado hacia otras personas, como son los hijos e hijas, padres y abuelos (Serret y Méndez 2011; 15). Empero, en el contexto pandémico, esta labor de cuidado ha incrementado, especialmente para las mujeres que tienen hijos o hijas, asumiendo las circunstancias actuales de educación en casa y el rol adicional de orientadoras educativas que tienen que desempeñar con más frecuencia las madres de familia (CEPAL-UNESCO, agosto de 2020).

Como sabemos, durante este periodo de contingencia sanitaria, el gobierno mexicano ha promovido la campaña «Quédate en casa» como una estrategia para evitar la 
propagación de la COVID-19 y proteger a la población del contagio, sin embargo, asumir el hogar como un sitio propio, natural y seguro para las mujeres resulta problemático si cuestionamos lo que este espacio ha implicado para muchas en tales condiciones. En ese sentido, el hogar parece representar un espacio más para la subordinación de las mujeres ya que, al ser un lugar atravesado por relaciones jerárquicas, no sólo persisten vínculos de dependencia económica y una recalcitrante exigencia social de hiper-productividad tras el confinamiento, sino subsisten otras formas de violencia indirecta que probablemente se basan en la culpa que genera el incumplimiento de los mandatos de género, por ejemplo, respecto a las labores domésticas 'propias de las mujeres'. Ello, sin adentrarnos en las cifras del tipo de violencia directa representadas en los altos niveles de violencia física contra las mujeres y tras el incremento de las agresiones sexuales a niñas, niños y adolescentes en los hogares de México (Este País, 13 de agosto de 2020).

En relación con el incremento de los niveles de violencia contra las mujeres durante la pandemia por SARS CoV-2, si bien la Organización Mundial de la Salud (OMS) reconoce que aún no existen muchos datos oficiales al respecto, lo cierto es que algunos informes de diferentes países del mundo, entre ellos China, Reino Unido y Estados Unidos de América, reportan que se ha producido un aumento considerable en el número de casos de violencia 
doméstica (Organización Mundial de la Salud, abril de 2020). En el caso concreto de México, el informe más reciente de Amnistía Internacional sobre la situación de los derechos humanos en el mundo señala que se agravó la situación de la violencia de género contra mujeres y niñas en razón de la COVID-19, la cual incluye actos de violencia de género intrafamiliar, violación, homicidio y feminicidio: «México fue uno de los países más afectados: en 2020 se habían registrado 3.752 homicidios de mujeres, de los cuales 969 se investigaron como feminicidios. Se preveía que las denuncias de incidentes de violencia contra las mujeres en el país superarían durante el año las 197.693 documentadas en 2019» (Amnistía Internacional 2020; 36).

Particularmente sobre violencia intrafamiliar en México, Amnistía Internacional reporta que: «Las llamadas al número de teléfono de emergencias 911 para denunciar actos de violencia contra las mujeres aumentaron durante el año, con 260.067 llamadas recibidas hasta diciembre, frente a un total de 197.693 en todo 2019» (Amnistía Internacional 2020; 307). Incluso según el informe sobre violencia contra las mujeres elaborado por el Sistema Nacional de Seguridad Pública de México, el cual contiene datos oficiales de las Fiscalías y Procuradurías de las 32 entidades federativas del país, es evidente un aumento de por lo menos $39.2 \%$ en los delitos de violencia de género en todas sus modalidades de acuerdo con su comparativo de enero de 2020 a enero de 
2021 (Secretaría de Seguridad y Protección Ciudadana 2021; 79), tal y como se muestra en el siguiente gráfico.

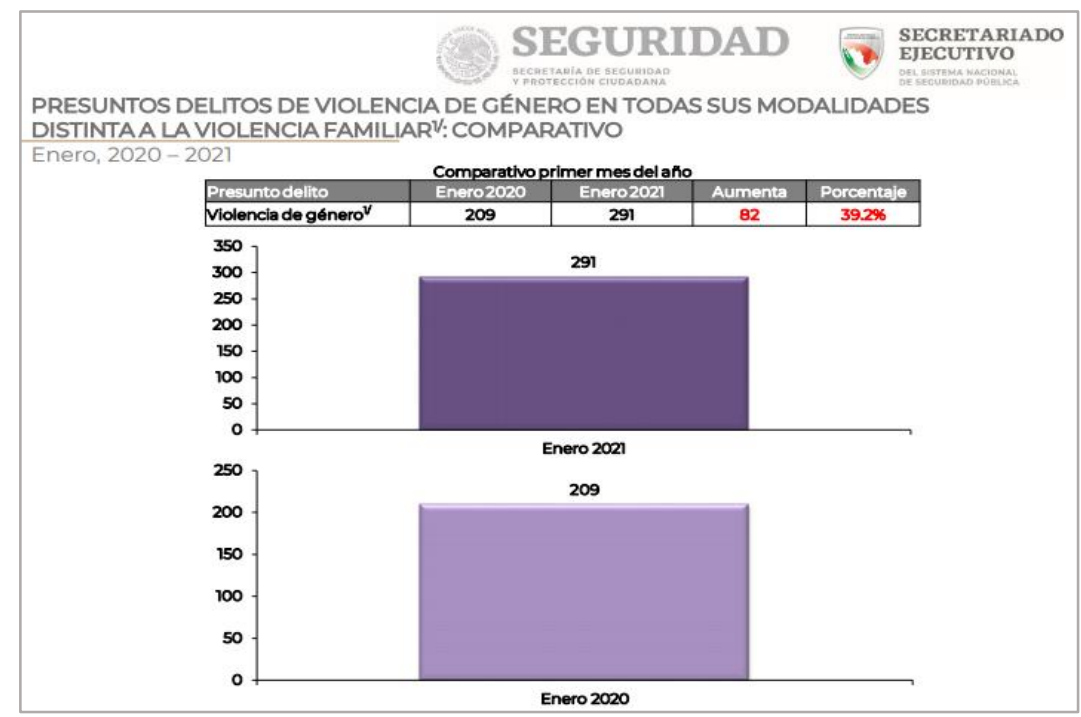

Recuperado de: Secretaría de Seguridad y Protección Ciudadana. (2021). Información sobre violencia contra las mujeres, México: Secretariado Ejecutivo del Sistema Nacional de Seguridad Pública, https://drive.google.com/file/d/1sXSQU6yy7r502TAFM_fW7mIVhGZIeVsm/view

Entre tanto, lo anterior nos invita a preguntarnos qué significa el «Quédate en casa». Si bien, aquella narrativa de la modernidad sobre lo público y lo privado nos estimulaba a pensar la casa como un espacio privado que permite la libertad personal, la seguridad, el confort y la salud, ahora en este contexto de confinamiento nos encaminamos más severamente a desidealizar el hogar como un espacio seguro para las mujeres en el que, siguiendo a la filósofa Sayak Valencia, tal parece que para las mujeres esta nueva 
normalidad representa una forma más de sumisión (COLEF, 16 de noviembre de 2020).

Anteriormente mencionábamos que la importancia de las teorías feministas de mediados del siglo $\mathrm{XX}$ es que han contribuido a que el uso del concepto y la categoría género empuje una metodología y un cambio epistémico relevante no sólo para las ciencias sociales sino para otros campos, lo cual poco a poco ha ido perfilando otra noción importante que es la denominada perspectiva de género. De acuerdo con Estela Serret y Jessica Méndez Mercado, la perspectiva de género es una forma de entender la realidad que considera las diferencias entre mujeres y hombres, así como sus posiciones desiguales en el mundo, las cuales se asumen como social y culturalmente construidas. En palabras de las autoras: «al aplicar la perspectiva de género, nuestra mirada de un fenómeno consigue: a) Visibilizar a las mujeres, sus actividades, sus vidas, sus necesidades específicas, sus espacios y la forma en que contribuyen a la creación de realidad social. b) Mostrar cómo y por qué cada fenómeno concreto está atravesado por las relaciones de poder y desigualdad entre los géneros características de los sistemas patriarcales o androcráticos» (Serret y Méndez 2012; 40).

Como puede apreciarse, si pudiéramos sintetizar las ventajas de aplicar la perspectiva de género, podríamos decir que se visibiliza a las mujeres en sus contextos concretos $\mathrm{y}$, además, se evidencia aquel legado que nos ha dejado la reformulación de la noción de género tras los 
aportes del pensamiento académico feminista al mostrar la forma en que las relaciones entre hombres y mujeres se han establecido desde la desigualdad y la asimetría de poder culturalmente consolidada en espacios públicos y privados.

Ahora bien, la perspectiva de género comienza a utilizarse dentro y fuera de la academia, lo cual fomenta el incluir una mirada feminista en muchas áreas, por ejemplo, en el poder político establecido, ya que ciertas instituciones han comenzado a tomar en cuenta estas elucubraciones para promover una cultura más justa para las personas, por ejemplo a través de políticas públicas incluyentes, así como exámenes sobre leyes y sistemas jurídicos con perspectiva de género buscando visibilizar y extinguir la discriminación y la violencia hacia las mujeres, no sólo en la redacción de los textos jurídicos sino en los efectos de las normas.

El análisis de género que cruza de forma transversal todos los campos de las ciencias humanas es utilizado para evaluar la participación política de las mujeres, así como las políticas públicas y sus objetivos. Y para ampliar el análisis político a esta forma transversal de reflexión, se le agrega la equidad y se corona con una perspectiva con equidad de género, que involucra el quehacer de hombres y mujeres (Dalton 2012; 84).

Sin embargo, en menester aclarar que la perspectiva de género no debe interpretarse de manera unívoca como un plan de acción acabado, por lo menos no en el terreno 
político, debido a que los gobiernos que pretendan implementarla deben analizar y determinar la forma en la que ésta se va incorporando de acuerdo al contexto social. En ese sentido, después de más de un año de confinamiento por COVID-19, es importante que desde las instancias gubernamentales se empiecen a fomentar políticas públicas enfocadas en atender este contexto específico de violencia contra las mujeres que se vive en los hogares. En otras palabras, desidealizar el hogar como un espacio seguro nos muestra que el confinamiento pandémico debería contar con aproximaciones y acciones con perspectiva de género, lo cual evidentemente no sólo involucra operaciones del gobierno mexicano, sino la conciencia social y la autocrítica respecto a cómo actuar empáticamente y con corresponsabilidad sobre las nuevas y viejas labores del hogar.

\section{Bibliografía}

Amnistía Internacional. (2020). Informe 2020/21 La situación de los derechos humanos en el mundo. Reino Unido: Amnesty International Ltd. Recuperado de: https://www.amnesty.org/download/Documents/PO L1032022021SPANISH.PDF 
Asuástegui Roig F. J., Rodríguez Uribes J.M., Peces-Barba Martínez G., y Fernández García E., (1998). Historia de los derechos fundamentales, Tomo I, España: Dikynson.

Conway, Jill K. et al. (2015). El concepto de género. En Lamas, M. (Comp.), El género La construcción cultural de la diferencia sexual (23-34), 2da Edición, México: Universidad Nacional Autónoma de México/Bonilla Artigas Editores.

Barya, N. (13 de agosto de 2020). Abuso sexual infantil: la otra pandemia. Revista Este País. Recuperado de https://estepais.com/sociedad_nueva/abuso-sexualinfantil-la-otra-pandemia/

Comisión Económica para América Latina y el Caribe/Organización de las Naciones Unidas para la Educación, la Ciencia y la Cultura. (2020). La educación en tiempos de la pandemia de COVID-19. Recuperado de https://repositorio.cepal.org/bitstream/handle/11362/ 45904/1/S2000510_es.pdf

Dalton, M. (2012). Democracia e igualdad en conflicto. México: Tribunal Electoral del Poder Judicial de la Federación / Centro de Investigaciones y Estudios Superiores en Antropología Social.

Delgado de Smith, Y. (2008). El sujeto: los espacios públicos $\mathrm{y}$ privados desde el género. Revista de Estudios 
Culturales, (2), 113-126. Recuperado de https://dialnet.unirioja.es/servlet/articulo?codigo=398 7106

El Colegio de la Frontera Norte. (2020). 25N Por la eliminación de la violencia contra las mujeres. Recuperado de https://www.colef.mx/noticia/25npor-la-eliminacion-de-la-violencia-contra-lasmujeres/

Fernández Poncela, A. (1998). Estudios sobre las mujeres, el género y el feminismo. Revista Nueva Antropología, (54), 79-95, México: Universidad Nacional Autónoma de México/Instituto de Investigaciones Jurídicas. Recuperado de https://revistascolaboracion.juridicas.unam.mx/index.php/nuevaantropologia/article/view/15761/14082

Galtung, J. (2003). Paz por medios pacífico, (trad. Victor A. León Gemmell), España: Bakeaz.

Hernández Islas, G. (27 de junio de 2019). Mexicanas: discriminación laboral, doble jornada y brecha salarial, Foro Jurídico. Recuperado de https://forojuridico.mx/mexicanas-discriminacionlaboral-doble-jornada-y-brecha-salarial/

Infobae, (12 de noviembre de 2020). El confinamiento por la pandemia de COVID-19 agudizó el abuso sexual infantil en México. Infobae. Recuperado de 
https://www.infobae.com/america/mexico/2020/11/12 /el-confinamiento-por-la-pandemia-de-covid-19agudizo-el-abuso-sexual-infantil-en-mexico/

Lamas, M. (2000). Diferencias de sexo, género y diferencia sexual. Revista Cuicuilco, 7(018), 1-25. Recuperado de https://www.redalyc.org/pdf/351/35101807.pdf

MacKinnon, C. (1987). Privacy v. Equality. En Feminism Unmodified: discourses of life and law, Cambridge/Harvard University Press.

Organización Mundial de la Salud (15 abril de 2020). Preguntas y respuestas: violencia contra la mujer durante la pandemia de COVID-19. Recuperado de: https://www.who.int/es/emergencies/diseases/novelcoronavirus-2019/question-and-answers-hub/q-adetail/violence-against-women-during-covid19?gclid=CjwKCAjw1uiEBhBzEiwAO9B_HdzGodxn KDE871G7N_FdKOiW9oiis-

GmITTAE86tXmGc2wredbZktBoCf_MQAvD_BwE

Romero, L. (20 de enero de 2020) Primer encuentro de comisiones internas de equidad de género. Gaceta UNAM. Recuperado de https://www.gaceta.unam.mx/primer-encuentro-decomisiones-internas-de-equidad-de-genero/

Saldarriaga Grisales, D.C. y Gómez Vélez, M.I. (2018). Teorías feministas, abolicionismo y decolonialidad: teorías críticas que cuestionan la efectividad de los 
derechos de las mujeres. Revista Prolegómenos Derechos y Valores, 21(41), 43-60, Recuperado de http://www.scielo.org.co/pdf/prole/v21n41/0121182X-prole-21-41-00043.pdf

Secretaría de Seguridad y Protección Ciudadana. (2021). Información sobre violencia contra las mujeres, México: Secretariado Ejecutivo del Sistema Nacional de Seguridad Pública. Recuperado de: https://drive.google.com/file/d/1sXSQU6yy7r502TAF M_fW7mIVhGZIeVsm/view

Serret, E. y Méndez Mercado, J. (2011). Sexo, género y feminismo, México: Suprema Corte de Justicia de la Nación.

Warner, M. (2012). Público, públicos y contrapúblicos, (trad. Victoria Schussheim), México: Fondo de Cultura Económica. 